\title{
Overview of the NASA Space Radiation Laboratory
}

\author{
Chiara La Tessa \\ Brookhaven National Laboratory, Upton 11973, NY, USA \\ Michael Sivertz \\ Brookhaven National Laboratory, Upton 11973, NY, USA \\ I-Hung Chiang $\dagger$ \\ Brookhaven National Laboratory, Upton 11973, NY, USA \\ Derek Lowenstein \\ Brookhaven National Laboratory, Upton 11973, NY, USA \\ Adam Rusek \\ Brookhaven National Laboratory, Upton 11973, NY, USA
}

\begin{abstract}
The NASA Space Radiation Laboratory (NSRL) is a multidisciplinary center for space radiation research funded by NASA and located at the Brookhaven National Laboratory (BNL), Upton NY. Operational since 2003, the scope of NSRL is to provide ion beams in support of the NASA Humans in Space program in radiobiology, physics and engineering to measure the risk and ameliorate the effect of radiation in space. Recently, it has also been recognized as the only facility in the U.S. currently capable of contributing to heavy ion radiotherapy research. This work contains a general overview of NSRL structure, capabilities and operation.
\end{abstract}

Keywords: Space radiation research, NASA

Email address: clatessa@bnl.gov (Chiara La Tessa)

$\dagger$ Deceased June $19^{\text {th }} 2016$.

Preprint submitted to Life Sciences in Space Research

October 8, 2016

(C) 2016. This manuscript version is made available under the Elsevier user license http://www.elsevier.com/open-access/userlicense/1.0/ 


\section{Introduction}

The increasing permanence of humans in Low Earth Orbit (LEO) and, in the future, in extended deep space missions beyond Earth, requires studies for assessing the risk from space radiation exposure and finding effective countermeasures. This has been the motivation for developing ground-based programs where the space radiation environment could be simulated.

The NASA Space Radiation Laboratory (NSRL) (Lowenstein and Rusek, 2007; Schimmerling, 2016) is a multidisciplinary accelerator-based center that provides charged particles for space radiation research. Commissioned in 2003, the facility is funded by NASA and managed in collaboration with Brookhaven National Laboratory (BNL), Upton NY, where it is located.

During its 13 years of operation, NSRL has served a large variety of experiments supported by the NASA Humans in Space program Miller and Zeitlin, 2016). The roadmap encompasses radiobiology studies for understanding the link between radiation exposure and carcinogenesis, Central Nervous System (CNS) damage, degenerative tissue, sterility and so on. These areas have been studied in acute and chronic exposure conditions to comprehend the short and long term effects of radiation on the astronauts' health. In addition, the optimization of shielding materials for dose mitigation, the issue of radiation sensitivity of electronics and nuclear physics measurements for model benchmarking have also been addressed in the program. Investigators unsupported by NASA have also been taking advantage of this facility, mostly researching topics common to NASA activities. Recently NSRL has been recognized as the only facility in the U.S. currently capable of contributing to heavy ion radiotherapy research (Held et al., 2016; Pompos et al., 2016). This has led to new interest in the facility and its capability from the medical community, creating opportunities to broaden the scope of activities outside the NASA interests.

Since the beginning of operations, NSRL has undergone many upgrades. As a result, three modes of operation are available: single species and monoenergetic beams, Solar Particle Event (SPE) simulator and Galactic Cosmic Ray (GCR) simulator.

The general features of NSRL are presented here. The overview will include a description of the beam line, details of the beam characteristics and delivery modes as well as the calibration procedures for various setups. 


\section{The facility}

\subsection{Beam line}

A schematic representation of the transport line from the ion source to NSRL exposure room is shown in Figure1 1 together with a map of the facility.

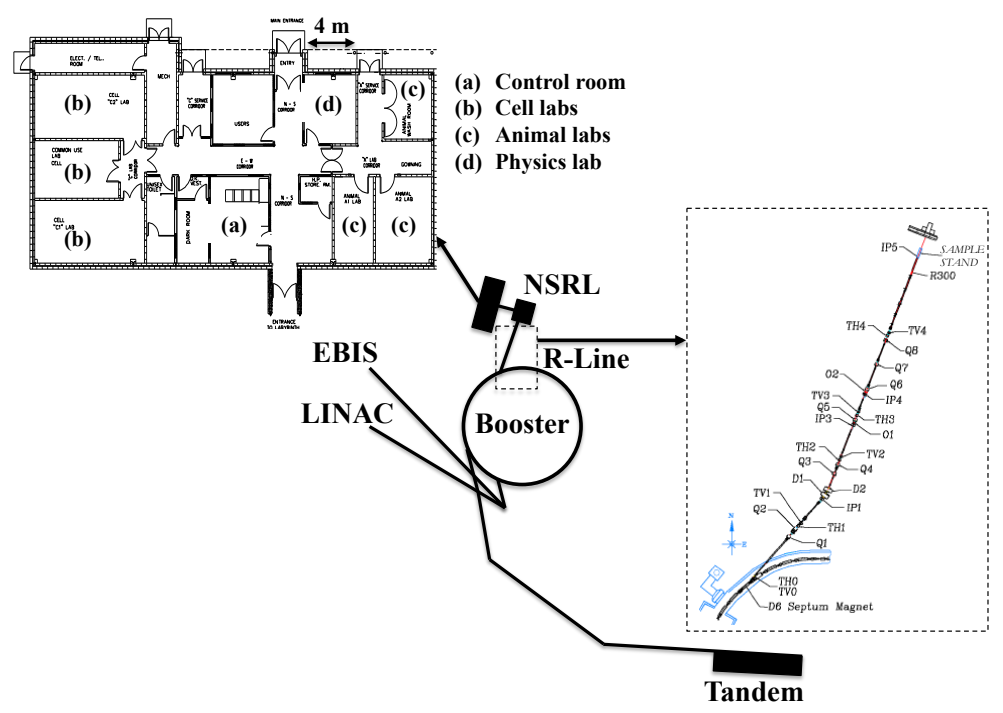

Figure 1: Layout of the accelerators and user facility for NSRL: sources (EBIS, Tandem and LINAC), synchrotron (Booster), transport segment between the Booster and the exposure room (R-Line) (Tsoupas et al., 2007) and NSRL building.

Three sources can provide ions to the Booster and ultimately to NSRL. These are the LINAC (protons only), the Tandem Van de Graaff (certain ions and protons), and the Electron Beam Ion Source (EBIS) (any ion except protons). Of these, the most used is the EBIS, now capable of selecting from a menu of solid source materials by use of Laser Ion Source (LIS) controlled by software which enables movement from target to target without expert assistance. The overall solid material ion switching can be done in one minute. EBIS is also equipped with two additional gas sources, which requires expert attention to change. Once all sources are in place, switching among them can be achieved within a minute. Current upgrade plans include the capability to accelerate protons from EBIS and preliminary tests have already been performed.

The particle beams produced by any of the sources mentioned above are injected into the Booster synchrotron where they are further accelerated to 
reach the final energy and delivered to NSRL via a single line branching off from the Booster (R-Line). A detailed description of all R-line elements is reported in (Tsoupas et al., 2007). The beam time structure can be varied over a large range from $300 \mathrm{~ms}$ to over $2 \mathrm{~s}$, with particles bunches (referred to as spills) delivered every $4-6 \mathrm{~s}$, depending on the other Booster activities.

Ion species from protons to gold are presently available, at energies ranging from $50 \mathrm{MeV} / \mathrm{u}$ to $2500 \mathrm{MeV}$ for protons and $1500 \mathrm{MeV} / \mathrm{u}$ for ${ }^{56} \mathrm{Fe}$. Lower energies can be achieved with passive absorbers. The range versus LET in water for a number of particle species and kinetic energies featured at NSRL is plotted in Figure 2.

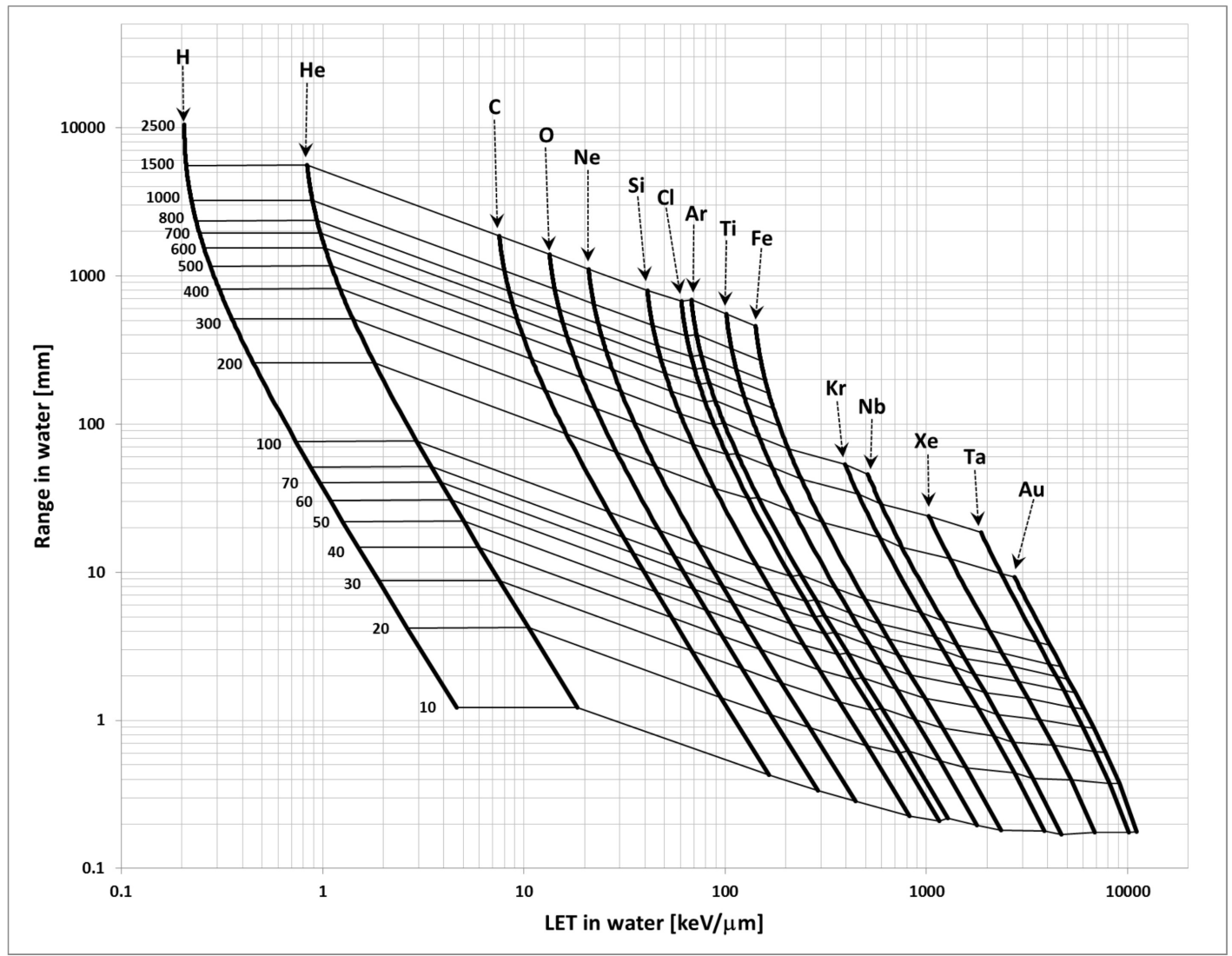

Figure 2: Range versus LET in water for several ion species and kinetic energies featured at NSRL. 


\subsection{Beam characteristics}

Beam shaping is done in the $100 \mathrm{~m}$ long transport segment between the Booster and NSRL irradiation room (R-Line). Since the facility was intended to accommodate radiobiology work as well as physics and engineering experiments, the R-line was designed to produce a large square beam spot with a uniform radiation field. The most common size used is $20 \mathrm{x} 20 \mathrm{~cm}^{2}$, although it can be made as large as $60 \times 60 \mathrm{~cm}^{2}$. This beam spot is achieved by use of a combination of dipole, quadrupole and octupole magnets, without any active beam scanning (Tsoupas et al., 2007). Examples of a pencil beam and the standard field are shown in Figures $3 \mathrm{a}$ and $3 \mathrm{~b}$, respectively.

Custom-shaped beams are achievable with the aid of collimators and shielding blocks of different sizes and materials (Figure 4).

Intensities available at NSRL are ion and energy dependent. Typical dose rates for the NASA work do not exceed $1 \mathrm{~Gy} / \mathrm{min}$ independent of the beam type. However, higher intensities can easily be achieved either by increasing the ion flux directly at the source or by reducing the field size. Lower values, down to approximately 100 particles $\mathrm{cm}^{-2}$, can be achieved for all ion species combining collimation and beam optics. This is done upstream of the R-Line to ensure beam purity and to preserve spatial uniformity at all intensities.

\section{3. $S P E$ and $G C R$ simulators}

Both SPE and GCR simulators aim at providing a radiation environment closer to what will be encountered on interplanetary missions.

The SPE simulator uses a proton beam with energies ranging from 50 to $150 \mathrm{MeV}$. The initial energy is set to the highest value and degraded with the binary filter. The fluence for each beam is modeled after two SPE events (1972 and 1989) to reproduce the dose profile in a human or, if appropriately scaled, in a small animal such a mouse or rat.

The GCR simulator (GCRSim) is designed to deliver a mixed radiation field consisting of different ions, from protons to Fe, at energies ranging from about $100 \mathrm{MeV} / \mathrm{u}$ to $1500 \mathrm{MeV} / \mathrm{u}$ for all ions, with protons going up to 2500 $\mathrm{MeV}$. This project required many upgrades and additions in both hardware and control-software to bring to fruition. The ability to rapidly and reliably switch ion species is achieved by use of the LIS (Section 2.1) . Controls were also upgraded to enable switching of the charge state produced by the EBIS and the energy to which the booster accelerates the beam, again without expert assistance. Ion and energy changes can now be achieved by a single command within less than two minutes. Furthermore, the original beam line 

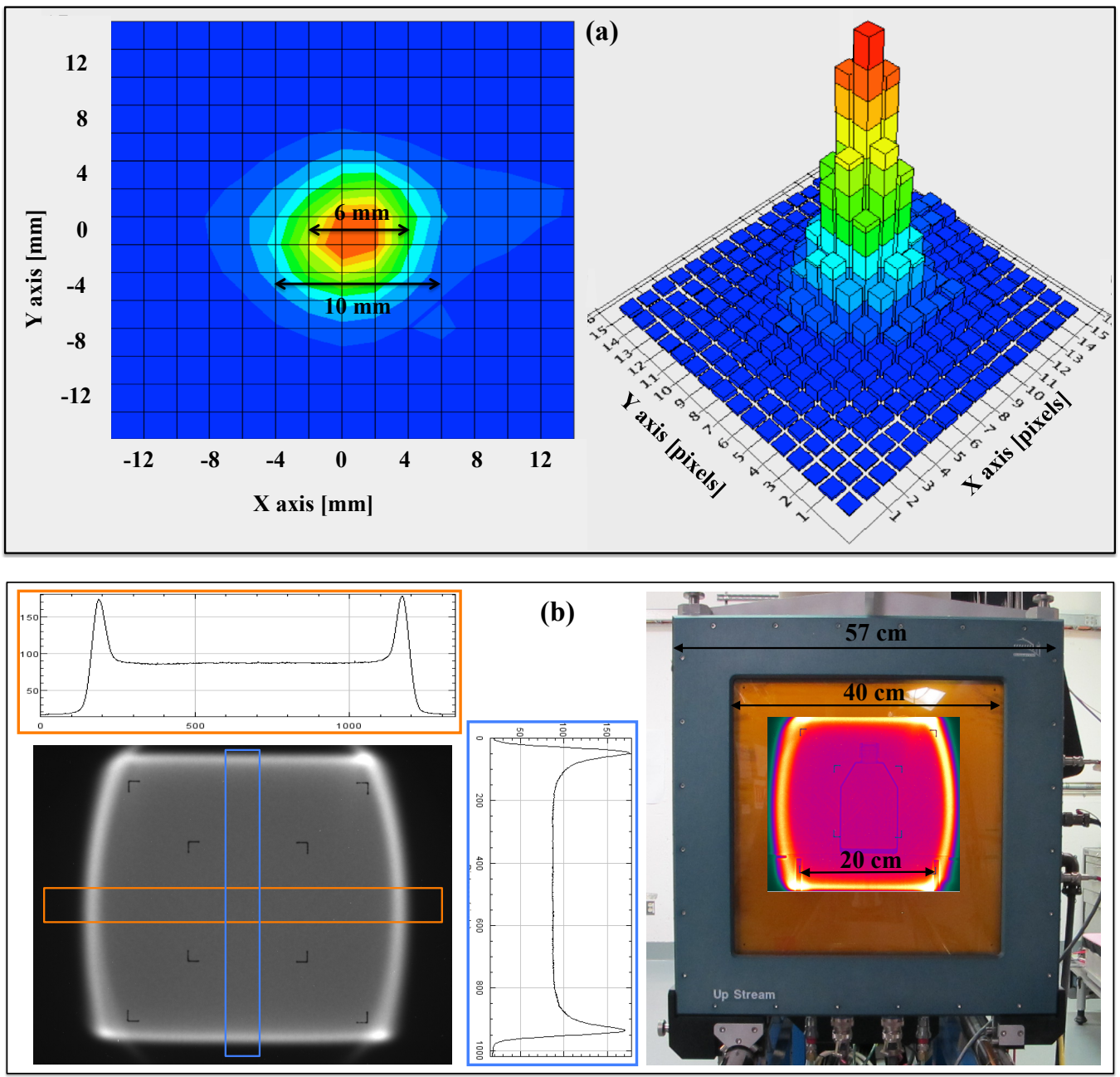

Figure 3: Typical profiles for a round beam (a) with $1 \mathrm{~cm}$ diameter and a 20x20 $\mathrm{cm}^{2}$ field (b). In panel (b), the right figure shows a typical $20 \times 20 \mathrm{~cm}^{2}$ beam overlaid on the image of one of the dosimetry ion chambers (Section 3) while the left figure presents a beam image acquired with the Digital Beam Imager (Section 3.2 and processed with ImageJ to obtain the horizontal and vertical profiles. 


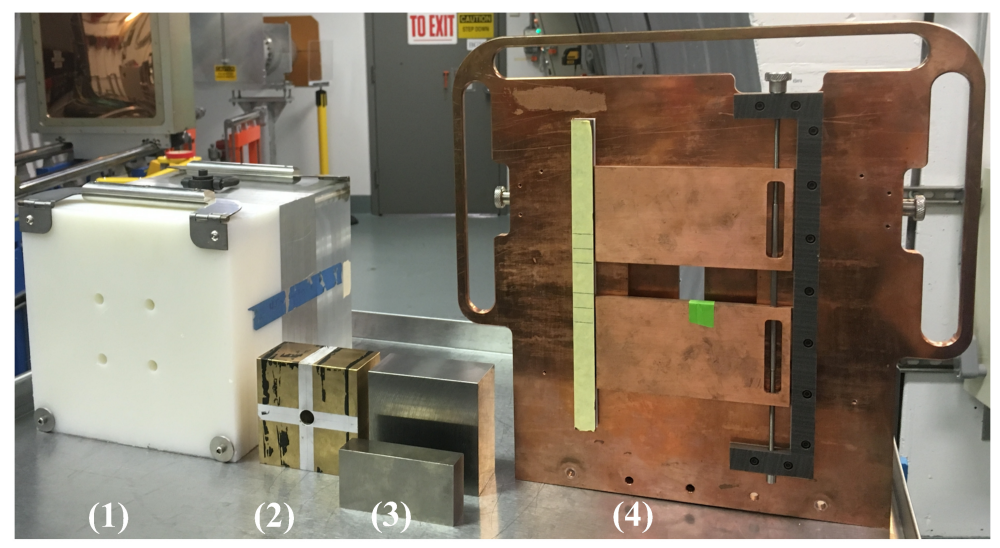

Figure 4: Collimators and shielding blocks available at NSRL for producing custom-shaped beams. (1) Composite three-layer collimators made of polyethylene, aluminum and lucite optimized for reducing the out-of-field dose to less than $2 \%$ when irradiated with a 600 $\mathrm{MeV} / \mathrm{u}^{56} \mathrm{Fe}$ beam. They have been used for other ions up to energies that would result in a background dose not above 3\%. Different sizes and hole configurations are available. (2) Brass collimators with round apertures between 14 and $18 \mathrm{~mm}$. They are used for reducing the beam size and halo as well as to produce parallel pencil beams. (3) Tungsten blocks of various dimensions with which various collimator shapes can be achieved. A combination of custom blocks resulted in an out-of-field dose of $2 \%$ when exposed to 1000 $\mathrm{MeV} / \mathrm{u}{ }^{4} \mathrm{He}$. (4) A copper collimator with adjustable size up to $10 \mathrm{x} 10 \mathrm{~cm}^{2}$, originally intended for shaping low-energy beams. 
design was limited to $1000 \mathrm{MeV} / \mathrm{u}$, so an upgrade to $1500 \mathrm{MeV} / \mathrm{u}$ was carried out, as required by the GCRSim specifications. This was accomplished by upgrading the extraction magnet to handle the higher currents needed for the higher fields, and the addition of a third magnet in the 20 degree bend. No new or additional power supplies were needed, though some repairs and upgrades to the extraction magnet power supply were performed. A variablethickness stripping-foil changer has also been installed at the upstream end of the extraction magnet, to improve control over the source-beam shape going into the R-Line. This is a very important factor, controlling the beam shape at the sample stand and, consequently, the validity of the calibration in going from one ion/energy combination to another.

Several strategies have been proposed to use the GCR simulator for reproducing space radiation fields in different scenarios. Details can be found in (Slaba et al., 2016; Kim et al., 2015) while a general review of the project including its impact on the NSRL scientific program is presented in (Kim et al., 2015).

While the GCRSim offers the possibility to deliver a custom mix of ions and energies within the available choices, two reference fields have been defined (Slaba et al., 2016). One approach (referred to as external field) approximates the external, free space GCR spectrum with discrete ions and energy beams and deliver them onto a shielding material placed in front of the sample. The absorber is used to modulate the incoming beams in a manner similar to vehicle or habitat shielding for a deep space mission. An alternative to the external field, called local tissue field approach, is to deliver a mix of beams that directly reproduce the dose profile in a representative tissue of an astronaut behind a shielding. Details on how to reproduce the external and local tissue fields are described in (Slaba et al., 2016). An example of the dose profile in a mouse-like phantom exposed to isotropic radiation and corresponding LET spectrum necessary to deliver it are shown in Figure 5 .

\section{Beam delivery and dosimetry}

\subsection{Ion species and energy}

Given a Booster magnetic rigidity and the ion species and charge state, it is possible to predict what energy the beam will have at the sample position. However, the beam type and energy are verified for each experiment. 


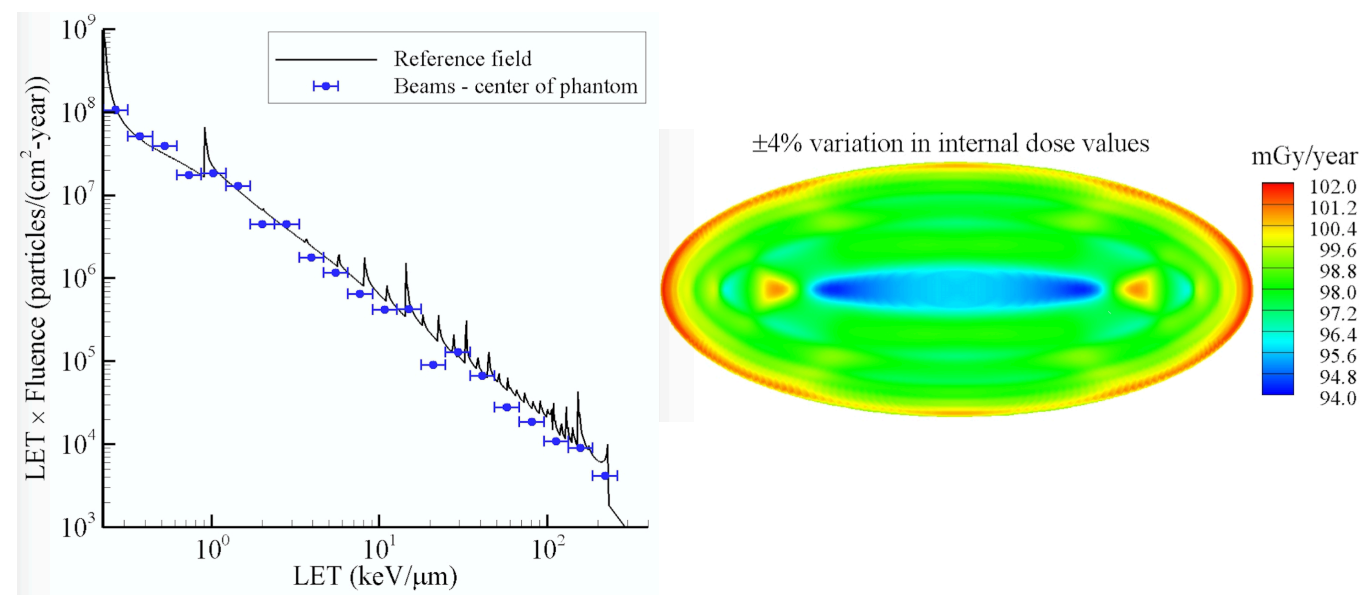

Figure 5: Dose profile in a mouse-like phantom (right) exposed to isotropic radiation and corresponding LET spectrum (left) necessary to deliver it. The latter is calculated using a continuous reference field (line) and discrete beams (dots). Figure taken from (Slaba et al. 2016).

Using the program LET ${ }^{2}$ (Zajic, 2004), the range of the selected ions in High Density Polyethylene (HDPE) is predicted. Upon performing a Bragg curve measurement using a binary filter made of HDPE, and checking the particles' range, we can be sure that the ion we are requesting is indeed the one delivered and the energy can be measured to greater precision at the sample position. Examples of Bragg curves for $205 \mathrm{MeV}$ protons, $293 \mathrm{MeV} / \mathrm{u}{ }^{12} \mathrm{C}$ ions and $963 \mathrm{MeV} / \mathrm{u}^{56} \mathrm{Fe}$ ions are shown in Figure 6.

Producing a Bragg curve uniquely identifies the beam characteristics and thus represents the best method to assure that the desired ion at the desired energy is being delivered.

\subsection{Field size and uniformity}

Beam images are acquired every spill with the Digital Beam Imager (DBI) shown in Figure 7. This detector, placed behind the sample stand, is triggered at the beginning of each spill and features a $40 \times 40 \mathrm{~cm}^{2}$ fluorescence screen and a pair of mirrors that acts as a periscope allowing the CCD camera (Hamamatsu ORCA-AG) to remain out of the direct beam. The light

\footnotetext{
${ }^{2}$ A comparison between LET and SRIM (Ziegler et al., 2010) programs resulted in negligible differences over the energy range used at NSRL.
} 


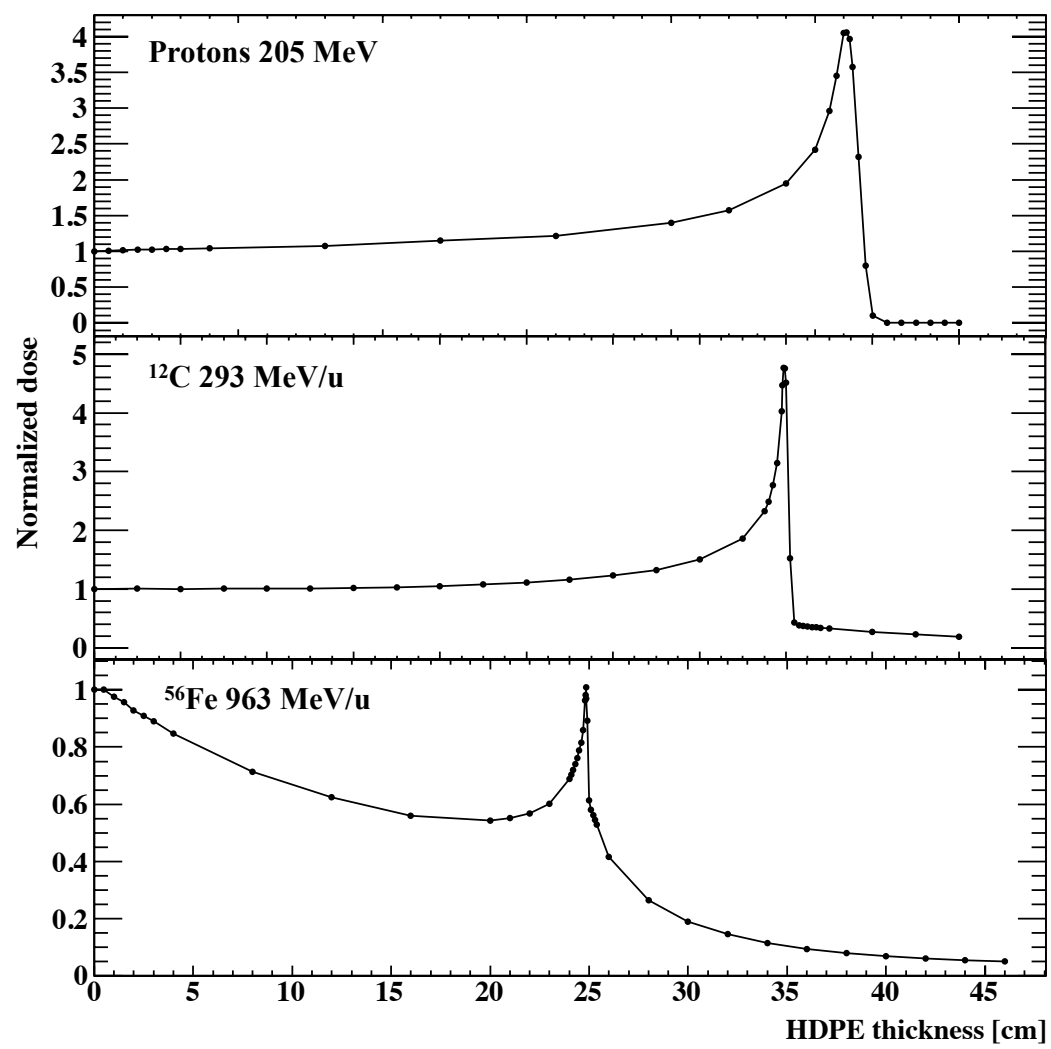

Figure 6: Bragg curves of $205 \mathrm{MeV}$ protons, $293 \mathrm{MeV} / \mathrm{u}{ }^{12} \mathrm{C}$ ions and $963 \mathrm{MeV} / \mathrm{u}{ }^{56} \mathrm{Fe}$ ions measured in High Density Polyethylene (HDPE). 
produced by the particles passing through the screen is redirected by the mirrors to the CCD, which collects it for the entire spill length and then produces an image.

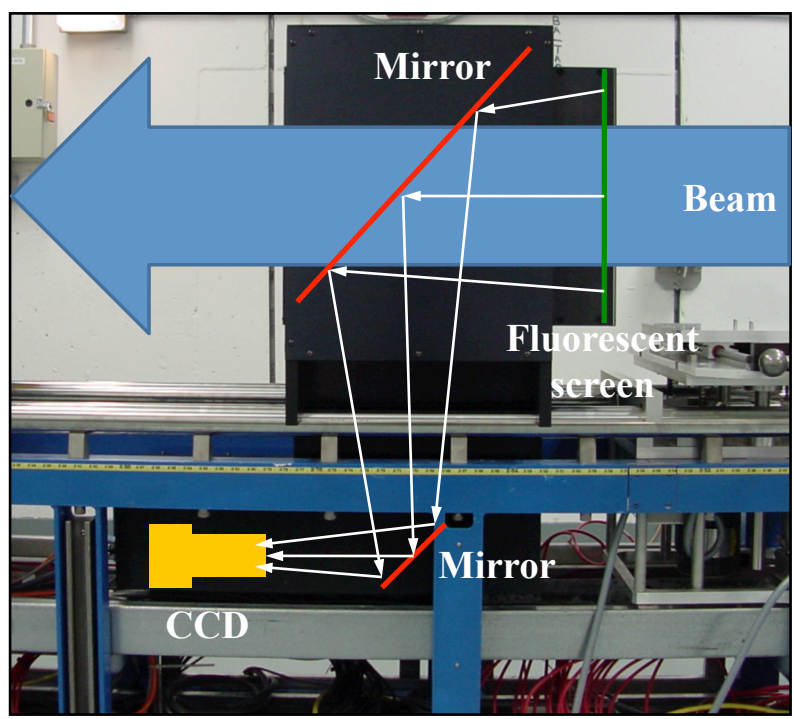

Figure 7: Scheme of the Digital Beam Imager (DBI).

With the help of the DBI, the beam size can be set at the beginning of each experiment, and checked continuously and adjusted during the exposures. Examples of images recorded by the Beam Imager are displayed in Figure $3 \mathrm{~b}$.

The DBI is also the main tool for assessing the beam uniformity. Saving a gray-scale image and analyzing it in ImageJ or equivalent software allows one to quantify the uniformity, as shown in the left picture of Figure $3 \mathrm{~b}$. The bright yellow frame at the edges of the beam field (right picture of Figure $3 \mathrm{~b}$ ) is translated into "ears" in both the horizontal and vertical profiles (left picture of Figure 3b). This feature is the result of the method used to create a large uniform beam, employing octupole magnets. As the outer frame has a higher dose than the uniform central field, it is never used for irradiations. We have learned from experience that independent of the particle type and energy a $3 \%$ uniformity can be achieved by eye.

\subsection{Dose calibration}

Calibration of dose is done against a NIST-traceable thimble ion chamber (referred to as the EGG counter), normally placed at the center of the beam. 
Almost all work at NSRL is carried out with a large, spatially uniform beam. In Figure $3 \mathrm{~b}$ an image of the dosimetry ion chamber is shown, overlaid with a beam image. The only material intercepted by the beam is the thin films in the ion chamber, never coming close to the ion chamber frame. The area used for dose measurement is completely contained within the uniform area of the beam. This makes the calibration both easy to perform, and much more reliable and meaningful over the entire uniform area of the beam (excluding the bright edges).

The availability of heavy ions at NSRL presents a unique opportunity to cross check the NIST-traceable ion chamber against scintillation counters. This chamber is calibrated with gamma radiation, the calibration given in coulombs per Roentgen. This number is converted to recycling integrator counts per Rad in water, using a method well-documented in the literature (ICRU, 1998), but is intended for work with protons only. With heavy ions one can use beams at low enough rate to read out both an ion chamber response and count individual ions in a $1 \mathrm{~cm}^{2}$ scintillation counter, thus tying together the dose measurement to the flux. With good enough control over beam shape and purity, which NSRL has, one can cross calibrate the thimble chamber. Such an effort was carried out at NSRL, using several ions including $\mathrm{C}$ and $\mathrm{Fe}$, establishing a $2 \%$ agreement between the NIST-traceable calibration chamber and the scintillation counter.

\subsection{Cutoff}

One of the three ion chambers located just upstream of the exposure stand can be chosen to be calibrated against the NIST-traceable thimble ion chamber, located at the point where the beam is to be delivered, and then used to measure the dose at that location during irradiation. The readout system and the associated irradiation control module can cut the accelerator beam off as soon as the desired dose is reached. The cutoff time is on the order of $1 \mathrm{~ms}$, representing a small fraction of a percent uncertainty on the integrated dose measurement. At low intensities, where the ion chamber is substituted by the scintillation counter, the cutoff is achieved with a countbased method.

\subsection{NSRL exposure room layout}

A sketch of the beam line downstream of the exit window in the NSRL exposure room is displayed in Figure 8. All elements are mounted on a 


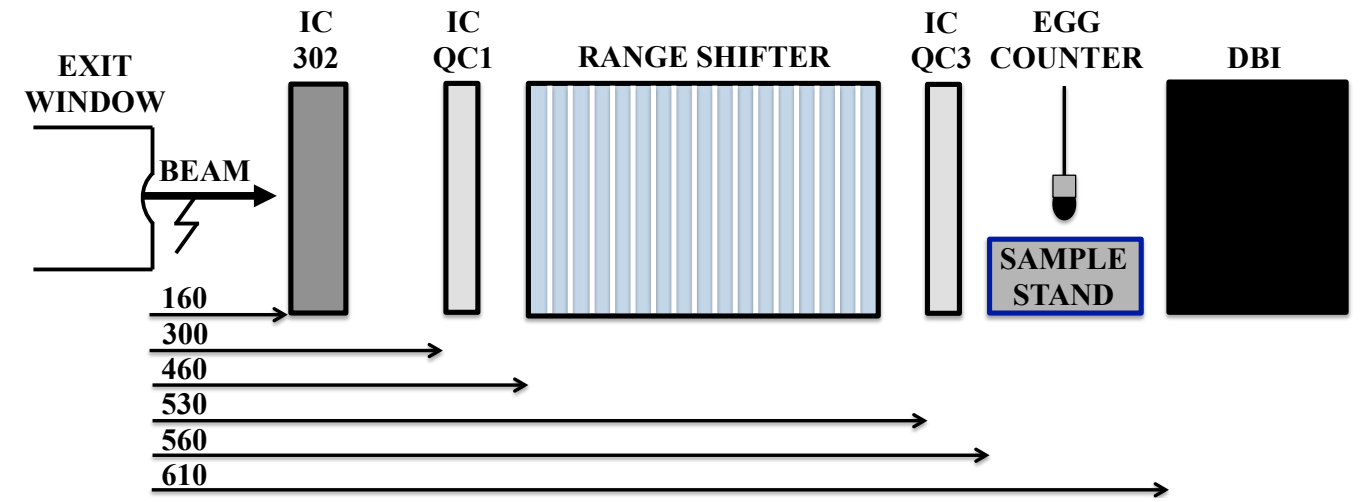

rail system and thus can be moved to accomodate different setups. Distances indicated in Figure 8 are typical of most experiments. Thicknesses of all elements are given in Table 1 and further details can be found in https://www.bnl.gov/nsrl/userguide/material-in-the-beam.php. Values for the range shifter and the EGG counter are not reported because they are removed from the beam path once the dose calibration and energy verification procedures are completed.

Figure 8: Layout of NSRL irradiation room. IC 302, QC1 and QC3 are Ionization Chambers. As all elements are movable, the distances can changed from setup to setup. The values reported are typical of most experiments and expressed in $\mathrm{cm}$.

Table 1: Thickness of all elements in the NSRL exposure room.

\begin{tabular}{cc}
\hline Element type & $\begin{array}{c}\text { Thickness } \\
\left(\mathrm{g} \mathrm{cm}^{-2}\right)\end{array}$ \\
\hline Exit window $(\mathrm{Al})$ & 0.103 \\
IC 302 & 0.123 \\
IC QC1 & 0.068 \\
IC QC3 & 0.068 \\
Air (20 degrees, 760 mm Hg) & 0.698 \\
\hline Total & 0.991 \\
\hline
\end{tabular}

\subsection{Non standard setups}

Two additional irradiation modes have been developed for certain experiments, especially those related to particle therapy: the stopping beam and 
the Spread Out Bragg Peak (SOBP).

In the former, the monoenergetic beam has to stop inside the sample or even at a specific depth, e.g. where the layer of cells is located. The challenge of this setup is to get an accurate measurement of the Bragg peak position, which can be achieved at a submillimeter precision with the DBI. Once the absorber thickness required for stopping the particles has been theoretically estimated, several images of the beam are acquired by adding or removing slabs of the binary filter to the initial calculated value. As the particles slow down in the degrader, the image brightness increases, reaches its maximum at the Bragg peak and then drops steeply, following the Bragg curve trend. Identifying the absorber thickness for which the image is brightest provides an accurate measurement of the beam stopping point. The calibration procedure for this setup cannot be accomplished with the standard methodology described in Section 3.3 but must be reckoned from dose measurements carried out by an ion chamber which intercepts the beam before it reaches the degrader, at a point where the energy is high enough to give a reliable and stable measurement.

NSRL has the capability of producing a Spread Out Bragg Peak with both an active change of the primary beam energy or by passive range modulation. Even if the process of changing energy is relatively fast (below $5 \mathrm{~min}$ ), it was never optimized as it was beyond the scope of the facility. Thus, the passive method with the use of a range shifter wheel is usually preferred. Typically, the wheel has a $1 \mathrm{~mm}$ step size as a narrower Bragg peak cannot be produced in the range of energies considered. This value takes into account the intrinsic energy spread of the beam in the Booster as well as the energy straggling in the absorber. For the dosimetry, the SOBP is considered as a combination of monoenergetic stopping beams, each calibrated according to the procedure explained above.

The high energy heavy ion beam provided at NSRL combined with the stopping beam mode allow Single Event Effects (SEE) testing of electronics without delidding the components. The ions can have a long enough range in silicon to completely penetrate the parts under test. This makes testing much simpler, avoiding the need for vacuum chambers and obviating a detailed map of the internal structure of the parts.

By using degraders of varying thickness, the Bragg peak can be scanned across the sensitive volume within the parts, studying the LET-dependence of upsets. LET scans typically begin at the highest beam energy (lowest LET) with the ions completely penetrating the device under test (DUT). By 
adding degrader to reduce the beam energy, it is possible to observe how upsets initially increase in number with increasing LET and then abruptly stop as the ions no longer have the energy required to reach the sensitive volume of the DUT. In this way, it is not necessary to have a detailed map of the internal structure of the DUT. Instead, the upset rate as a function of degrader thickness can be used to make a maps of the internal structure.

This Variable Depth Bragg Peak (VDBP) Method (Buchner et al., 2011) has been proven to be a powerful tool in electronics testing. The variable degrader allows for a simple and quick measurement of the effective thickness of parts. Placing the DBI behind the parts to be tested allows for a measurement of the range of the ion beam in polyethylene with and without the part in the beam, immediately giving the polyethylene equivalent thickness of the part.

The large beam spot allows for tests of entire systems of electronics. Some sensitivities to radiation do not show up at the part level, giving evidence only at the system level. The highly penetrating ions and large sample area allows complete circuit boards to be tested at once.

\subsection{Overall dose uncertainty}

Contributors to the overall dose uncertainty are beam purity, beam uniformity, calibration, readout electronics, cutoff. Of these, the dominant ones are beam uniformity $(3 \%)$ and calibration $(2 \%)$. Combining these in quadrature yields an overall dose uncertainty of $3.6 \%$.

\section{Support}

NSRL is staffed with several scientists and technicians at all times during running hours. Technical support is available for planning and designing experiments at NSRL, including engineering, design and fabrication of sample holders, special degraders and collimators. User-driven ideas and designs have led to many improvements and additions to NSRL's capabilities, and are welcomed.

For biology work there is a fully staffed laboratory complex in the BNL Biology Department, where lab space and support are available, as well as a fully AAALAC ${ }^{3}$ accredited animal facility (BLAF, Brookhaven Laboratory

\footnotetext{
${ }^{3}$ Association for Assessment and Accreditation of Lab Animal Care.
} 
Animal Facility). Personnel from both the biology support team and the BLAF are present at NSRL to support irradiation time involving their areas of responsibility.

For non-biology work, support is offered for both planning and carrying out of measurements or experiments.

In general, at least one NSRL physicist is present for all irradiations to ensure beam and dosimetry quality, interact with the main control room, and provide the oversight for smooth and safe operation.

\section{Summary}

For over a decade, researchers have been making use of the NSRL to conduct studies of the effects of space radiation on biological and electronic samples in an effort to reduce the risk of space flight on humans. During that time, NSRL has increased its capabilities and flexibility to better simulate the radiation environment of space. With the addition of the EBIS, changes in ion species and energy can be accomplished in a matter of minutes. The recent upgrade in beam energy enables NSRL to cover a greater part of the galactic cosmic ray spectrum. Tools like the SPE Simulator have been developed to meet the needs of researchers.

The large beam spot makes it possible to expose large numbers of samples at once, increasing the throughput and efficiency. Precise control of the beam cutoff, under the control of the experimenters, makes for accurate dose delivery.

Good uniformity of the radiation field intensity also contributes to reduce uncertainties in the delivered dose. The dose calibration is maintained through regular verification of the performance of the ion chambers used in the dose measurement. Cross checking the calibration using ion counting techniques validates the entire calibration procedure.

NSRL is currently the only facility in the world which combines all the following features:

- all ions relevant for space radioprotection and ion therapy available in the energy range of interest (up to $2500 \mathrm{MeV}$ for protons and 1500 $\mathrm{MeV} / \mathrm{u}$ for ${ }^{56} \mathrm{Fe}$ particles and below);

- fast switching of ion species and energy;

- SPE and GCR simlators; 
- large uniformly-distributed beam suitable for biology work as well as physics and engineering testing;

- dose-based cutoff achieved with a dedicated dosimetry system;

- high-quality spill by spill beam monitoring;

- animal facility.

Unlike other facilities, which are limited in ion selection and energy range, have to schedule around the main customers (as medical centers) or cannot accomodate animal work, NSRL does not have any of these constrains. It has been designed to maximize flexibility in the exposure area, labs and scheduling with room for upgrades arising from experience gained over the years of operation. As an example, in response to the growing interest in heavy ion therapy in United States, NSRL has been recognized as the only place where basics studies can be performed. Its role can be viewed as complementary to future ion therapy facilities, supporting all the experiments where clinical conditions are not required.

The goal of the NSRL is to provide a tool that can accurately simulate the radiation environment of space, that experimenters can use without concerns about the accuracy or reproducibility of the delivered dose. We continue to work towards that goal.

\section{Acknowledgements}

The work at NSRL is supported by NASA (Contract No. T570X) and performed under the United State Department of Energy (Contract No. DEAC02-98CH10886). The authors would like to thank the Collider Accelerator Department main control room staff and the operations support technicians without whom the facility could not run smoothly. We wish to acknowledge the Ion Source EBIS personnel for the tremendous effort and extremely efficient work. A special thanks goes to NSLR chief engineer Charlie Pearson who endeavors to improve the facility every day.

\section{References}

Buchner, S., Kanyogoro, N., McMorrow, D., Foster, C., O'Neill, P., Nguyen, K., 2011. Variable depth Bragg peak method for single event effects testing. IEEE Transactions on Nuclear Science 58, 2976-2982. 
Held, K., Blakely, E., Story, M., Lowenstein, D., 2016. Use of the NASA Space Radiation Laboratory at Brookhaven National Laboratory to conduct charged particle radiobiology studies relevant to ion therapy. Radiat. Reas. 185, 563-567.

ICRU, 1998. Clinical proton dosimetry: Part I. Beam production, beam delivery, and measurement of absorbed dose. International Commission on Radiological Units and Measurements 59.

Kim, M.-H. Y., Rusek, A., Cucinotta, F. A., 2015. Issues for simulation of galactic cosmic ray exposures for radiobiological research at ground-based accelerators. Frontiers in Oncology 5, 122-.

URL http://journal.frontiersin.org/article/10.3389/fonc. 2015.00122

Lowenstein, D., Rusek, A., 2007. Technical developments at the NASA Space Radiation Laboratory. Radiat. Environ. Biophys. 46, 91-94.

Miller, J., Zeitlin, C., 2016. Twenty years of space radiation physics at the BNL AGS and NASA Space Radiation Laboratory. Life Sci. Space Res. 9, $12-18$.

Pompos, A., Durante, M., Choy, H., 2016. Heavy ions in cancer therapy. JAMA Oncol., 1-2.

Schimmerling, W., 2016. Genesis of the NASA Space Radiation Laboratory. Life Sci. Space Res. 9, 2-11.

Slaba, T., Blattnig, S., Norbury, J., Rusek, A., La Tessa, C., 2016. Reference field specification and preliminary beam selection strategy for acceleratorbased GCR simulation. Life Sci. Space Res. 8, 52-67.

Tsoupas, N., Ahrens, L., Bellavia, S., Bonati, R., Brown, K., Chiang, I.-H., Gardner, C., Gassner, D., Jao, S., Mackay, W., Marneris, I., Meng, W., Phillips, D., Pile, P., Prigl, R., Rusek, A., Snydstrup, L., Zeno, K., 2007. Uniform beam distributions at the target of the NASA Space Radiation Laboratory's beam line. Phys. Rev. ST Accel. Beams 10, 024701-024712.

Zajic, V., 2004. LET124. Http://tvdg10.phy.bnl.gov/LETCalc.html. 
382 Ziegler, J. F., Ziegler, M. D., Biersack, J. P., Jun. 2010. SRIM - The stopping and range of ions in matter (2010). Nuclear Instruments and Methods in 384 Physics Research B 268, 1818-1823. 